\title{
Uso de fitoterápicos no tratamento da dislipidemia: Um estudo de revisão
}

\author{
Herbal medicine use on dyslipidemia treatment: A review study \\ Uso de medicamentos a base de hierbas en el tratamiento de la dislipidemia: Un estudio de revision
}

Rayzza Marcelly Jesus Da Silva ORCID: https://orcid.org/0000-0002-1469-2293

Universidade Federal do Pará, Brasil E-mail: raymsilva14@gmail.com

Carliane Cardoso Dos Reis

ORCID: https://orcid.org/0000-0002-8156-3520 Universidade Federal do Pará, Brasil E-mail: reiscarli1@gmail.com

Matheus Lopes Cardoso ORCID: https://orcid.org/0000-0002-4893-8335 Universidade Federal do Pará, Brasil E-mail: Matheuscardosom@gmail.com

Jamille De Araujo Matos ORCID: https://orcid.org/0000-0003-3614-5335 Universidade Federal do Pará, Brasil

E-mail: jamillearaujomatos97@gmail.com

Enayle Rayane Silva Madeira ORCID: https://orcid.org/0000-0003-0106-4375 Universidade Federal do Pará, Brasil E-mail: enaylesilva.m@gmail.com

Victória Elizabeth Pinheiro Ferreira ORCID: https://orcid.org/0000-0003-2393-5557 Universidade Federal do Pará, Brasil E-mail: Vicelizabethpf15@gmail.com Marcela De Souza Figueira ORCID: https://orcid.org/0000-0002-3046-5242 Universidade Federal do Pará, Brasil E-mail:msfigueira@ufpa.br

\begin{abstract}
Resumo
Objetivo: analisar os efeitos de fitoterápicos no tratamento das dislipidemias. Metodologia: trata-se de uma revisão de literatura com pesquisas nos idiomas inglês, português e espanhol realizadas entre 2017 e 2021 disponíveis nas bases de dados PubMed, Scielo e Google Acadêmico. As pesquisas elegidas limitaram-se a seres humanos, sendo classificadas de acordo com seu nível de evidência. Resultados: os estudos apontaram a eficácia do Allium sativum L. (alho) na redução do colesterol total (CT) e do colesterol da lipoproteína de baixa densidade (LDL-c), além de promover ação antitrombótica e auxiliar na redução da agregação plaquetária. Linum usitatissimun L. (linhaça), nas formas de semente, pó, biscoito e óleo, demonstrou benefícios na redução dos níveis de CT, LDL-c, lipoproteína de alta densidade (HDLc) e triglicerídeos (TG) devido ao seu teor de fibras e ômega-3. Avena sativa (Aveia) também apresentou resultados positivos na redução nos níveis de TG, LDL-c e não-HDL-c por conter alto teor de fibras solúveis do tipo beta-glucana. Conclusão: os fitoterápicos analisados apresentaram resultados satisfatórios para a redução e controle das dislipidemias por atuarem no equilíbrio do teor de lipídeos circulantes.
\end{abstract}

Palavras-chave: Fitoterapia; Dislipidemias; Metabolismo dos lipídeos.

\begin{abstract}
Objective: analyze the effects based on herbal medicine on dyslipidemia treatment. Methodology: this literature review is on English, Portuguese, and Spanish used searches between 2017 and 2021 available on databases PubMed, Scielo, and Google Scholar. The research was limited to human beings, according to its evidence level. Results: the studies point to the Allium sativum L. (garlic) effectiveness use to reduces total cholesterol (TC), low-density lipoprotein (LDLc) in addition to reducing antithrombotic action and platelet aggregation. The Linum usitatissimun L. (linseed), in seed shape of powder, biscuit, and oil, shows those benefits on reductions level of TC, LDL-c, high-density lipoprotein (HDL-c), and triglycerides (TG) due to its fiber omega-3 content. The Avena sativa (oat) also shows positive results on reduction levels TG, LDL-c, and non-HDL-c because of its high content of beta-glucan-type fibers. Conclusion: the herbal medicines analyzed presented great results from reduction and dyslipidemia control due to their balance on circulating lipids.
\end{abstract}

Keywords: Herbal medicine; Dyslipidemia; Lipid metabolism. 


\begin{abstract}
Resumen
Objetivo: analizar los efectos de las hierbas medicinales en el tratamiento de las dislipidemias. Metodología: se trata de una revisión bibliográfica con investigación en inglés, portugués y español realizada entre 2017 y 2021 disponible en las bases de datos PubMed, Scielo y Google Scholar. Las investigaciones elegidas se limitaron a los seres humanos y se clasificaron de acuerdo con su nivel de evidencia. Resultados: los estudios señalaron la eficacia de Allium sativum L. (ajo) en la reducción del colesterol total (CT) y del colesterol de lipoproteínas de baja densidad (LDL-c), además de promover una acción antitrombótica y ayudar a reducir la agregación plaquetaria. Linum usitatissimun L. (linaza), en forma de semilla, polvo, galleta y aceite, demostró beneficios en la reducción de los niveles de CT, LDL-c, lipoproteína de alta densidad (HDL-c) y triglicéridos (TG) debido a su contenido en fibra y omega-3. Avena sativa (Avena) también demostró resultados positivos en la reducción de los niveles de TG, LDL-c y noHDL-c por poseer un alto contenido de fibras solubles de tipo beta-glucano. Conclusión: los fitoterapéuticos analizados presentaron resultados satisfactorios para la reducción y control de las dislipidemias porque actúan en el equilibrio del contenido lipídico circulante.
\end{abstract}

Palabras clave: Fitoterapia; Dislipidemias; Metabolismo dos lípidos.

\title{
1. Introdução
}

Ao longo do tempo, a cura através de plantas medicinais foi evoluindo tradicionalmente de forma cumulativa, e, a partir disso, construiu-se tratamentos para diversas doenças (Santana, Sá, Neves, Figueredo, \& Viana, 2018). A fitoterapia é definida como uma terapêutica de origem vegetal que usa plantas medicinais de diferentes formas farmacêuticas, porém, sem o uso de substâncias isoladas (Ministério da Saúde, 2006).

Um dos marcos da fitoterapia foi a Portaria $n^{\circ} 3916 / 98$ que aprovou a Política Nacional de Medicamentos que estabeleceu seu uso no âmbito científico e tecnológico. Em 1996, o relatório da $10^{\circ}$ Conferência Nacional de Saúde predispõe a incorporação não só da fitoterapia, mas também da acupuntura e da homeopatia, que confirmava a prática de terapias alternativas e populares. Além disso, a Resolução no 338/04 do Conselho Nacional de Saúde aprovou a Política Nacional de Assistência Farmacêutica dispondo do uso de plantas medicinais e de medicamentos fitoterápicos no âmbito da atenção à saúde, propondo o respeito do conhecimento tradicional e científico direcionado à adoção de políticas de emprego e renda (Ministério da Saúde, 2006).

Por outro lado, as dislipidemias são anormalidades no metabolismo dos lipídios, apresentando em quantidades alteradas no plasma e podendo elevar o risco de doenças cardiovasculares. Ela pode ser atrelada a baixos níveis de colesterol de lipoproteína de alta densidade (HDL-c), a altos níveis de triglicerídeos (TG), colesterol total (CT), e colesterol de lipoproteína de baixa densidade (LDL-c), o que pode contribuir para a aterosclerose, doença arterial coronariana e hipertensão arterial sistêmica (He, et al., 2018; Précoma, et al., 2019; Souza, et al., 2019)

De acordo com sua etiologia, a dislipidemia pode ser dividida em dois grupos, primárias e secundária, onde a primeira tem sua origem genética e a segunda é causada por influência externa, como uso de medicamentos, estilo de vida inadequado e outras doenças, como Hepatopatia Crônica, Diabetes mellitus tipo II, Insuficiência renal crônica e síndrome nefrótica, obesidade, bulimia e anorexia, tabagismo, etilismo, medicamentos betabloqueadores, diuréticos, corticosteroides, anabolizantes, anticoncepcionais, entre outros. Além disso, de acordo com a sua classificação laboratorial, pode ser dividida em 4 grupos: Hipercolesterolemia isolada (aumento isolado de LDL-c, onde LDL-c $\geq 160 \mathrm{mg} / \mathrm{dl}$ ), Hipertrigliceridemia isolada (aumento isolado de triglicérides, onde $\mathrm{TG} \geq 150 \mathrm{mg} / \mathrm{dl}$ em jejum), Hiperlipidemia mista (LDL-c e TG elevados) e HDL-c baixo (homens $<40 \mathrm{mg} / \mathrm{dl}$ e mulheres < $50 \mathrm{mg} / \mathrm{dl}$ ). (Précoma, et al., 2019; Nogueira-de-Almeida, et al., 2017)

A população mundial sofreu uma transição nutricional em que há a presença de ingestão elevada de produtos industrializados cuja base são açúcares simples, gorduras e sódio. Os medicamentos fitoterápicos também são usados para o tratamento das dislipidemias, uma vez que eles têm credibilidade popular e são de baixo custo. Além disso, eles são capazes de diminuir altas concentrações de lipídios, principalmente aqueles marcadores de distúrbios metabólicos e de dislipidemias. (Bezerra, Oliveira, Vilar, Confessor, \& Vilar, 2017).

A partir dos dados da Vigilância de Fatores de Risco e Proteção para Doenças Crônicas por Inquérito Telefônico 
(VIGITEL) de 2016 (Ministério da Saúde, 2017) ao averiguar 26 capitais brasileiras e o Distrito Federal, indicou-se uma frequência de $24,8 \%$ de indivíduos que relataram ser diagnosticados com dislipidemias. Nessa perspectiva, estudos transversais com base populacional brasileira com o público-alvo composta por infanto-juvenis e adultos apontaram uma prevalência de 22,6\% e de 49,7\% de dislipidemias nos respectivos grupos (Camargo, Zamarchi, Balieiro, Pessoa, \& Camargo, 2021; Teixeira, et al., 2021), expressando o contingente significativo acerca dos distúrbios metabólicos dos lipídios, além de enfatizar um problema no tocante à saúde pública, visto que o Ministério da Saúde indica que o Sistema Único de Saúde (SUS) destina em média 1,3 bilhão de reais por ano na terapêutica dessas alterações lipídicas (Oliveira, Carvalho, Dourado, Dourado, \& Nascimento, 2017).

A descompensação metabólica das dislipidemias contribui para a evolução de doenças cardiovasculares (Borges, et al., 2021), pois o desequilíbrio de lipídeos na corrente sanguínea pode resultar em processos sistêmicos que culminam na formação de placas ateroscleróticas, as quais estão envolvidas na fisiopatologia de doenças e eventos cardiovasculares, tais como infarto agudo do miocárdio, acidente vascular cerebral, doença cardíaca isquêmica, insuficiência cardíaca, doença arterial coronariana, doença cerebrovascular, doença vascular periférica, dentre outros.

A hipercolesterolemia isolada é o fator de risco mais importante para doença arterial coronariana (Précoma, et al., 2019). O aumento da concentração de LDL-c está relacionada a uma dieta hiperlipídica, com destaque às gorduras saturadas, e à maior expressão da síntese endógena de colesterol.

Além da concentração, o tamanho da molécula de LDL também é um fator importante para o desenvolvimento de doenças cardiovasculares, haja vista que a subclasse LDL-sd, que apresenta densidade reduzida em relação às demais, é capaz de invadir o endotélio vascular mais facilmente, além de estar mais suscetível à oxidação por radicais livre e à fagocitose por macrófagos com colesterol esterificado, possibilitando a formação de células espumosas e, consequentemente, de estrias lipídicas as quais são lesões primárias da formação da placa aterosclerótica. (Raymond \& Couch, 2018; Evangelista, Leite, \& Lima, 2013)

Concentrações reduzidas de HDL-c também é um fator de risco aterosclerótico, visto que essa molécula realiza o transporte reverso de colesterol circulante, além de proteger a molécula LDL do processo oxidativo. (Evangelista, Leite, \& Lima, 2013)

Nesse contexto, é válido ressaltar que o tratamento das dislipidemias com a fitoterapia promove o uso da biodiversidade vegetal e seu uso vinculado ao conhecimento tradicional e a tecnologia para a sua validação (Ministério da Saúde, 2006). Portanto, este estudo objetiva analisar os efeitos de fitoterápicos no tratamento das dislipidemias.

\section{Metodologia}

Este artigo trata-se de uma revisão narrativa da literatura de caráter descritivo, a respeito dos fitoterápicos usados no tratamento das dislipidemias. A base metodológica utilizada para este artigo foi a revisão bibliográfica narrativa descrita por Elias e colaboradores (2012) e Rother (2007) como sendo um método que possibilita a construção de artigos nos quais os autores possam elaborar análises e interpretações de forma mais abrangente e crítica, sob um ponto de vista teórico. Rother (2007) ressalta a necessidade de se limitar em fontes de qualidade para que haja uma análise fundamentada e profunda das informações.

A investigação da literatura para base teórica foi feita a partir da análise das três bases de dados: PubMed, Scielo, e Google Acadêmico. Na identificação das publicações relevantes, buscou-se nas bases de dados estudos realizados nos últimos cinco anos (2017 a 2021), aplicando os termos "Dislipidemias", “fitoterapia", “doença cardiovascular”, “perfil lipídico", "tratamento", "Herbal medicine" e "dyslipidemia".

Para a avaliação de inclusão, utilizou-se: artigos publicados nos idiomas espanhol, inglês e português, além das pesquisas limitarem-se aos seres humanos. Outrossim, para o processo de exclusão, retirou-se: Comunicações breves, artigos de 
revisão que não apresentavam sua versão na íntegra, trabalhos em duplicatas, pesquisas utilizando animais e estudos com mais de cinco anos, exceto estudos clássicos, como diretrizes e livros.

Os conteúdos foram ponderados quanto à principal questão da pesquisa: “fitoterápicos podem ser utilizados no tratamento das dislipidemias”. Em seguida, efetuou-se a leitura do título e do resumo. Após, os estudos foram marcados como incluídos e excluídos de acordo com os critérios estabelecidos para esses fins. Por fim, as pesquisas incluídas foram categorizadas de acordo com o nível de evidência conforme estabelecido por Melnyk e Fineout-Overholt (2018).

No fluxograma exposto (Erro! Fonte de referência não encontrada.), consta a quantidade de artigos encontrados, quantos os excluídos conforme os critérios de exclusão (comunicações breves, revisões em o texto na íntegra, estudos em duplicatas, pesquisa com o uso de animais e estudos com mais de 5 anos) para que fosse possível compor o quadro da revisão de literatura e fundamentar a discussão. A apuração dos artigos seguidas por uma triagem, foi relevante à fidedignidade do conteúdo, bem como para credibilizar a conclusão do artigo.

Figura 1 - Fluxograma de para seleção dos estudos.

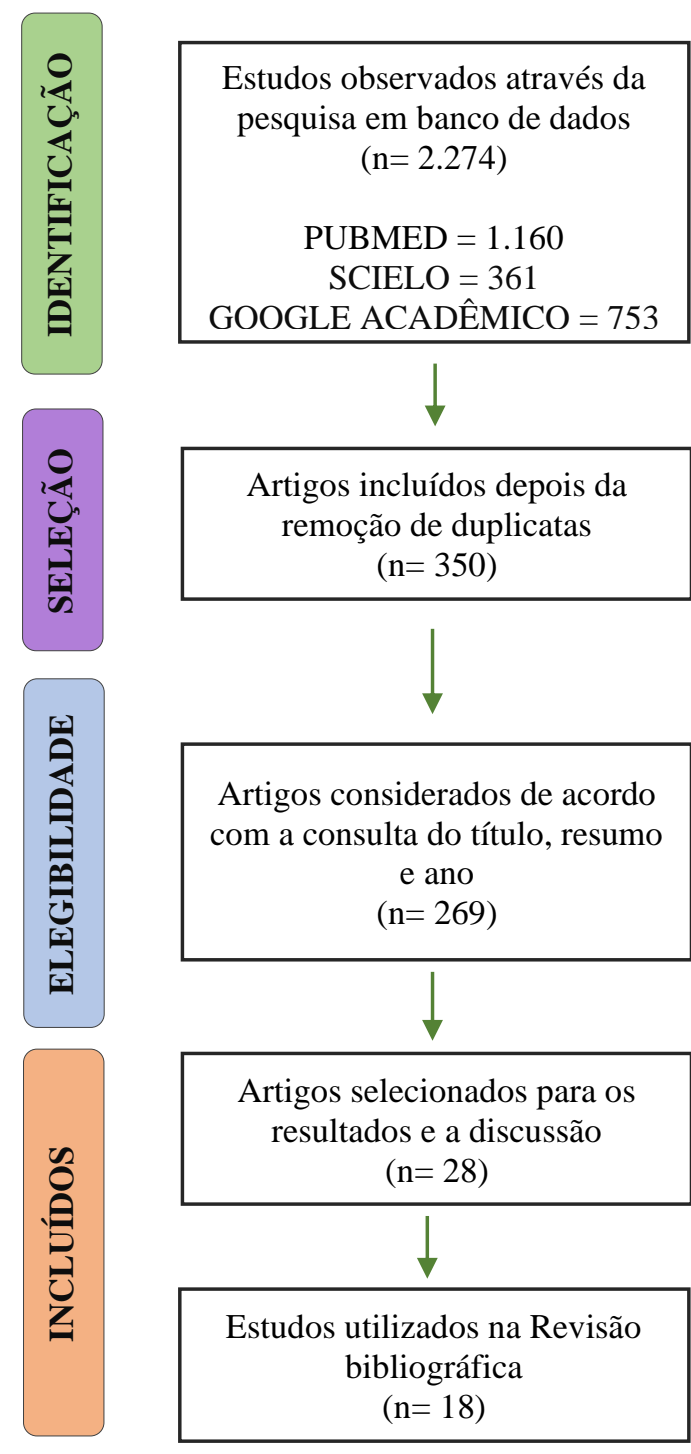

Fonte: Autores.

Artigos excluídos por título e resumo $(\mathrm{n}=1.924)$

Comunicações breves $=560$

Artigos de revisão sem texto na íntegra $=285$

Estudos realizados em animais $=305$

Trabalhos com mais de 5 anos $=774$ 


\section{Resultados}

Com base nos descritores citados para a execução dessa revisão, estimou-se um total de 2.274 artigos, nos quais 269 foram considerados conforme a consulta do resumo, título e ano. Nesse viés, observou-se e utilizou-se 28 pesquisas, para obter os resultados e embasar a discussão, as quais obedeceram a relação do fitoterápico com as dislipidemias.

Em concordância com os critérios de inclusão, os 18 estudos elegidos foram contemplados por intermédio da leitura completa com o intuito de compreender e retirar os principais levantamentos e características da pesquisa as quais aparecem incluso no Quadro 1.

Quadro 1 - Exposição bibliográfica encontrada nas bases de dados.

\begin{tabular}{|c|c|c|c|c|}
\hline Título do artigo & $\begin{array}{c}\text { Ano de } \\
\text { publicação }\end{array}$ & Tipo de estudo & Objetivo & Principais achados \\
\hline $\begin{array}{l}\text { Effect of raw crushed garlic } \\
\text { (allium sativum l.) On } \\
\text { components of metabolic } \\
\text { syndrome (Choudhary, Jani, \& } \\
\text { Sharma, 2018) }\end{array}$ & 2018 & $\begin{array}{l}\text { ensaio clínico } \\
\text { randomizado } \\
\text { (nível de } \\
\text { evidência 2) }\end{array}$ & $\begin{array}{l}\text { investigar o efeito } \\
\text { do alho cru } \\
\text { esmagado sobre os } \\
\text { componentes da } \\
\text { síndrome } \\
\text { metabólica }\end{array}$ & $\begin{array}{c}\text { o alho amassado cru foi capaz de diminuir } \\
\text { a circunferência da cintura, pressão } \\
\text { arterial sistólica e diastólica, } \\
\text { triglicerídeos, glicose no sangue em jejum } \\
\text { e, ainda, aumentar o nível colesterol } \\
\text { sérico de lipoproteína de alta densidade } \\
\text { (HDL) }\end{array}$ \\
\hline $\begin{array}{l}\text { Aged garlic extract preserves } \\
\text { cutaneous microcirculation in } \\
\text { patients with increased risk for } \\
\text { cardiovascular diseases: A } \\
\text { double-blinded placebo- } \\
\text { controlled study (Wlosinska M. } \\
\text {, et al., 2019) }\end{array}$ & 2019 & $\begin{array}{l}\text { estudo } \\
\text { randomizado } \\
\text { duplo-cego } \\
\text { controlado por } \\
\text { placebo (nível de } \\
\text { evidência 2) }\end{array}$ & $\begin{array}{l}\text { avaliar o efeito do } \\
\text { tratamento de longo } \\
\text { prazo com extrato } \\
\text { de alho envelhecido } \\
\text { na perfusão do } \\
\text { tecido cutâneo }\end{array}$ & $\begin{array}{c}\text { percebeu-se que durante o tempo de } \\
\text { estudo os indivíduos que passaram por } \\
\text { uma intervenção ao utilizar o suplemento } \\
\text { de extrato de alho era capazes de } \\
\text { preservar e melhorar a microcirculação } \\
\text { cutânea }\end{array}$ \\
\hline $\begin{array}{l}\text { Aged garlic extract reduces il- } \\
\text { 6: a double-blind placebo- } \\
\text { controlled trial in females with } \\
\text { a low risk of cardiovascular } \\
\text { disease (Wlosinska M. , et al., } \\
\text { 2021) }\end{array}$ & 2021 & $\begin{array}{l}\text { estudo } \\
\text { randomizado } \\
\text { duplo-cego } \\
\text { controlado por } \\
\text { placebo (nível de } \\
\text { evidência 2) }\end{array}$ & $\begin{array}{c}\text { avaliar se a } \\
\text { suplementação } \\
\text { diária de extrato de } \\
\text { alho envelhecido } \\
\text { (AGE) poderia } \\
\text { reduzir a inflamação } \\
\text { em mulheres com } \\
\text { baixo risco de } \\
\text { doença } \\
\text { cardiovascular } \\
\end{array}$ & $\begin{array}{l}\text { mulheres que sofreram intervenção ao } \\
\text { usar o extrato de alho envelhecido } \\
\text { tiveram níveis mais baixos de marcador } \\
\text { inflamatório IL-6, bem como a redução } \\
\text { do perfil lipídico ao comparar mulheres } \\
\text { que utilizaram o placebo no período de } 12 \\
\text { meses }\end{array}$ \\
\hline $\begin{array}{c}\text { A systematic review and meta- } \\
\text { analysis of ayurvedic herbal } \\
\text { preparations for } \\
\text { hypercholesterolemia (Gyawali, } \\
\text { Vohra, Orme-Johnson, } \\
\text { Ramaratnam, \& Schneider, } \\
\text { 2021) }\end{array}$ & 2021 & $\begin{array}{l}\text { ensaio clínico } \\
\text { randomizado } \\
\text { (nível de } \\
\text { evidência 2) }\end{array}$ & $\begin{array}{l}\text { explorar a força das } \\
\text { evidências sobre a } \\
\text { eficácia e segurança } \\
\text { das ervas para } \\
\text { hipercolesterolemia }\end{array}$ & $\begin{array}{l}\text { a meta-análise dos ensaios mostrou que o } \\
\text { alho reduzir o LDL-c em } 10,37 \mathrm{mg} / \mathrm{dL} \\
(95 \% \text { CI }-17,58 \text { a }-3,16 ; \text { p-valor }=0,005) \\
\text { e que é eficaz para reduzir } \\
\text { moderadamente à hipercolesterolemia }\end{array}$ \\
\hline $\begin{array}{c}\text { Estudio experimental y } \\
\text { controlado en farmacia } \\
\text { comunitaria: influencia de la } \\
\text { administración de un } \\
\text { complemento alimenticio de ajo } \\
\text { en la evolución de la } \\
\text { hipercolesterolemia (Suances, } \\
\text { Estévez, \& Estévez, 2018) }\end{array}$ & 2018 & $\begin{array}{l}\text { ensaio clínico } \\
\text { controlado (nível } \\
\text { de evidência 2) }\end{array}$ & $\begin{array}{l}\text { estudar a variação } \\
\text { dos valores do } \\
\text { colesterol total em } \\
\text { pacientes tratados } \\
\text { com suplemento } \\
\text { alimentar à base de } \\
\text { alho em cápsulas }\end{array}$ & $\begin{array}{l}\text { observou-se que o efeito do suplemento } \\
\text { alimentar de alho não parece ser eficaz o } \\
\text { suficiente para reduzir significativamente } \\
\text { os níveis de colesterol }\end{array}$ \\
\hline $\begin{array}{c}\text { Therapeutic effects of garlic in } \\
\text { cardiovascular atherosclerotic } \\
\text { disease (Sobenin, Myasoedova, } \\
\text { Iltchuk, Zhang, \& Orekhov, } \\
\text { 2019) }\end{array}$ & 2019 & $\begin{array}{c}\text { revisão } \\
\text { sistemática (nível } \\
\text { de evidência 1) }\end{array}$ & $\begin{array}{l}\text { avaliar o nível de } \\
\text { evidência } \\
\text { disponível para os } \\
\text { diferentes efeitos } \\
\text { protetores do alho e } \\
\text { compreender os } \\
\text { mecanismos } \\
\text { subjacentes } \\
\end{array}$ & $\begin{array}{l}\text { a revisão mostrou evidências de que os } \\
\text { efeitos benéficos do alho em diferentes } \\
\text { aspectos dos distúrbios cardiovasculares, } \\
\text { como as dislipidemias, permaneceram } \\
\text { insuficientes para traçar recomendações } \\
\text { precisas, embora o consenso permaneça } \\
\text { favorável ao uso do alho }\end{array}$ \\
\hline
\end{tabular}




\begin{tabular}{|c|c|c|c|c|}
\hline $\begin{array}{c}\text { Linseed oil increases HDL3 } \\
\text { cholesterol and decreases blood } \\
\text { pressure in patients diagnosed } \\
\text { with mild } \\
\text { hypercholesterolaemia } \\
\text { (Skoczyńska, Gluza, } \\
\text { Wojakowska, Turczyn, \& } \\
\text { Skoczyńska, 2018) }\end{array}$ & 2018 & $\begin{array}{l}\text { ensaio clínico } \\
\text { aberto (nível de } \\
\text { evidência 2) }\end{array}$ & $\begin{array}{l}\text { avaliar o efeito da } \\
\text { suplementação } \\
\text { alimentar de curto } \\
\text { prazo com óleo de } \\
\text { linhaça sobre a } \\
\text { pressão arterial } \\
\text { (PA) e o } \\
\text { metabolismo } \\
\text { lipídico em } \\
\text { pacientes com } \\
\text { hipercolesterolemia } \\
\text { leve } \\
\end{array}$ & $\begin{array}{l}\text { a suplementação com óleo de linhaça } \\
\text { diminuiu significativamente o colesterol } \\
\text { de lipoproteína de baixa densidade (LDL) } \\
\text { e lipoproteína de não alta densidade, e } \\
\text { aumentou os níveis de colesterol HDL e } \\
\text { HDL3. Além disso, o óleo de linhaça } \\
\text { reduziu a PA diastólica em homens, nas } \\
\text { mulheres o óleo de linhaça reduziu a PA } \\
\text { sistólica e a PA diastólica }\end{array}$ \\
\hline $\begin{array}{l}\text { Effects of flaxseed on blood } \\
\text { lipids in healthy and } \\
\text { dyslipidemic subjects: a } \\
\text { systematic review and meta- } \\
\text { analysis of randomized } \\
\text { controlled trials (Masjedi, Pour, } \\
\text { Shokoohinia, \& Asgary, 2021) }\end{array}$ & 2021 & $\begin{array}{c}\text { revisão sistemática } \\
\text { e meta-análise de } \\
\text { ensaios clínicos } \\
\text { randomizados (nível } \\
\text { de evidência 1) }\end{array}$ & $\begin{array}{l}\text { determinar o efeito } \\
\text { da semente de } \\
\text { linhaça no perfil } \\
\text { lipídico (CT, LDL- } \\
\text { C, HDL-C e TGs) } \\
\text { de indivíduos } \\
\text { saudáveis em } \\
\text { comparação com } \\
\text { dislipidêmicos }\end{array}$ & $\begin{array}{l}\text { os suplementos de linhaça promovem } \\
\text { uma melhora notável no perfil lipídico de } \\
\text { indivíduos dislipidêmicos e indivíduos } \\
\text { saudáveis. Notou-se alteração importante } \\
\text { nos níveis de CT, LDL-C e TGs de } \\
\text { indivíduos dislipidêmicos, sem alteração } \\
\text { no HDL-C. Em indivíduos saudáveis, } \\
\text { houve alteração nos níveis de CT, LDL-C } \\
\text { e HDL-C, sem alteração no TGs }\end{array}$ \\
\hline $\begin{array}{l}\text { Effect of flaxseed or psyllium } \\
\text { vs. placebo on management of } \\
\text { constipation, weight, glycemia, } \\
\text { and lipids: a randomized trial } \\
\text { in constipated patients with type } \\
2 \text { diabetes (Soltanian \& } \\
\text { Janghorbani, 2019) }\end{array}$ & 2018 & $\begin{array}{l}\text { ensaio clínico } \\
\text { simples-cego, } \\
\text { randomizado e } \\
\text { controlado por } \\
\text { placebo. (nível de } \\
\text { evidência 2) }\end{array}$ & $\begin{array}{l}\text { comparar os } \\
\text { sintomas de } \\
\text { constipação, peso, } \\
\text { glicemia e lipídios } \\
\text { em pacientes } \\
\text { constipados com } \\
\text { diabetes tipo } 2 \\
\quad \text { (T2D) que } \\
\text { receberam linhaça } \\
\text { assada ou psyllium } \\
\text { versus aqueles que } \\
\text { receberam placebo }\end{array}$ & $\begin{array}{l}\text { apesar de tanto a linhaça quanto o } \\
\text { psyllium possam reduzir os sintomas de } \\
\text { constipação, peso, níveis glicêmicos e } \\
\text { lipídicos, o tratamento com linhaça } \\
\text { apresenta ser superior ao psyllium }\end{array}$ \\
\hline $\begin{array}{c}\text { Effect of flaxseed powder on } \\
\text { cardiovascular risk factor in } \\
\text { dyslipidemic and hypertensive } \\
\text { patients (Haghighatsiar, Askari, } \\
\text { Saraf-Bank, Feizi, \& Keshmiri, } \\
\text { 2019) }\end{array}$ & 2019 & $\begin{array}{l}\text { ensaio clínico } \\
\text { randomizado } \\
\text { duplo-cego } \\
\text { controlado por } \\
\text { placebo. (nível de } \\
\text { evidência 2) }\end{array}$ & $\begin{array}{c}\text { avaliar os efeitos do } \\
\text { sachê de linhaça } \\
\text { sobre os níveis } \\
\text { séricos de perfis } \\
\text { lipídicos, pressão } \\
\text { arterial, índices } \\
\text { antropométricos e } \\
\text { açúcar no sangue } \\
\text { em jejum em } \\
\text { pacientes } \\
\text { hipertensos } \\
\text { dislipidêmicos } \\
\end{array}$ & $\begin{array}{c}\text { partir dos efeitos benéficos da linhaça } \\
\text { acerca dos fatores de risco cardiovascular, } \\
\text { a utilização da linhaça pode ser apontada } \\
\text { como um procedimento terapêutico útil } \\
\text { para diminuir o perfil lipídico e os índices } \\
\text { antropométricos. }\end{array}$ \\
\hline $\begin{array}{c}\text { Effect of flaxseed } \\
\text { supplementation on lipid } \\
\text { profile: an updated systematic } \\
\text { review and dose-response meta- } \\
\text { analysis of sixty-two } \\
\text { randomized controlled trials } \\
\text { (Hadi, et al., 2020) }\end{array}$ & 2019 & $\begin{array}{l}\text { revisão sistemática } \\
\text { atualizada e meta- } \\
\text { análise de dose- } \\
\text { resposta de sessenta } \\
\text { e dois ensaios } \\
\text { randomizados } \\
\text { controlados. (nível } \\
\text { de evidência 1) }\end{array}$ & $\begin{array}{c}\text { revisar } \\
\text { sistematicamente as } \\
\text { evidências atuais } \\
\text { para identificar os } \\
\text { efeitos potenciais da } \\
\text { suplementação de } \\
\text { linhaça nos perfis } \\
\text { de lipídios do } \\
\text { sangue usando uma } \\
\text { meta-análise de } \\
\text { ensaios controlados }\end{array}$ & $\begin{array}{c}\text { ingerir linhaça e seus produtos têm uma } \\
\text { consequência benéfica na diminuição de } \\
\text { TC, LDL-C e TG em humanos e seria } \\
\text { capaz de propiciar um plano alternativo } \\
\text { para controlar dislipidemias e doenças } \\
\text { cardiovasculares }\end{array}$ \\
\hline $\begin{array}{l}\text { Flaxseed (Linum usitatissimum } \\
\text { l.) supplementation in patients } \\
\text { undergoing lipoprotein } \\
\text { apheresis for severe } \\
\text { hyperlipidemia-a pilot study } \\
\text { (Kanikowska, et al., 2020) }\end{array}$ & 2020 & $\begin{array}{l}\text { estudo clínico } \\
\text { piloto, } \\
\text { unicêntrico, } \\
\text { prospectivo (nível } \\
\text { de evidência 3) }\end{array}$ & $\begin{array}{c}\text { determinar se a } \\
\text { suplementação de } \\
\text { linhaça pode ajudar } \\
\text { a controlar o } \\
\text { colesterol no sangue } \\
\text { e reduzir a carga } \\
\text { inflamatória em } \\
\text { pacientes que } \\
\text { necessitam de } \\
\text { aférese de } \\
\text { lipoproteína }\end{array}$ & $\begin{array}{l}\text { a suplementação de linhaça produziu } \\
\text { efeito consistente de redução do } \\
\text { colesterol e do LDL no grupo intervenção } \\
\text { em comparação ao grupo placebo }\end{array}$ \\
\hline
\end{tabular}




\begin{tabular}{|c|c|c|c|c|}
\hline $\begin{array}{l}\text { The role of specific components } \\
\text { of a plant-based diet in } \\
\text { management of dyslipidemia } \\
\text { and the impact on } \\
\text { cardiovascular risk (Trautwein } \\
\text { \& McKay, 2020) }\end{array}$ & 2020 & $\begin{array}{l}\text { revisão Narrativa } \\
\text { (nível de } \\
\text { evidência 5) }\end{array}$ & $\begin{array}{c}\text { sumarizar o } \\
\text { conhecimento atual } \\
\text { dos compostos a } \\
\text { base de plantas na } \\
\text { redução do lipídio e } \\
\text { colesterol (LDL-C) } \\
\text { sanguíneo, assim } \\
\text { como de doenças } \\
\text { cardiovasculares }\end{array}$ & $\begin{array}{l}\text { o resumo demonstrou que alguns } \\
\text { compostos presentes nas plantas, em } \\
\text { especial a beta-glucana da aveia, são } \\
\text { capazes de reduzir, de forma significativa, } \\
\text { o LDL-C e não-HDL-C, auxiliando na } \\
\text { prevenção de doenças cardiovasculares }\end{array}$ \\
\hline $\begin{array}{c}\text { Efecto de la mezcla de cereales } \\
\text { con extracto acuoso de Ananás } \\
\text { comosus en los niveles séricos } \\
\text { de colesterol y triglicéridos de } \\
\text { personas con dislipidemia } \\
\text { (Medina, 2017) }\end{array}$ & 2017 & $\begin{array}{l}\text { ensaio clínico } \\
\text { controlado (nível } \\
\text { de evidência 2) }\end{array}$ & $\begin{array}{c}\text { determinar o efeito } \\
\text { que possui a mistura } \\
\text { de cereais } \\
\text { juntamente com o } \\
\text { extrato bruto de } \\
\text { Ananás comosus em } \\
\text { pessoas com } \\
\text { dislipidemia no } \\
\text { distrito de Chiclayo } \\
\text { Lambayeque } \\
\end{array}$ & $\begin{array}{l}\text { observou-se que o extrato, juntamente } \\
\text { com os cereais, possui efeito } \\
\text { hipolipemiante em seres vivos. }\end{array}$ \\
\hline $\begin{array}{c}\text { A randomized placebo- } \\
\text { controlled clinical trial to } \\
\text { evaluate the medium-term } \\
\text { effects of oat fibers on human } \\
\text { health: the beta-glucan effects } \\
\text { on lipid profile, glycemia and } \\
\text { intestinal health (belt) study } \\
\text { (Cicero, et al., 2020) }\end{array}$ & 2020 & $\begin{array}{l}\text { ensaio clínico } \\
\text { controlado (nível } \\
\text { de evidência 2) }\end{array}$ & $\begin{array}{l}\text { analisar os efeitos } \\
\text { da beta-glucana, } \\
\text { derivada da aveia, } \\
\text { na redução de LDL- } \\
\text { C, não-HDL-C e } \\
\text { colesteróis totais, de } \\
\text { humanos. }\end{array}$ & $\begin{array}{l}\text { comprovou-se que a suplementação de } \\
3 \mathrm{~g} / \text { dia de beta-glucana, possui certa } \\
\text { eficácia na redução de LDL-C, TC e não- } \\
\text { HDL-C em pessoas com } \\
\text { hipercolesterolemia leve. }\end{array}$ \\
\hline $\begin{array}{c}\text { Oat bran, but not its isolated } \\
\text { bioactive } \beta \text {-glucans or } \\
\text { polyphenols, have a bifidogenic } \\
\text { effect in an in vitro fermentation } \\
\text { model of the gut microbiota } \\
\text { (Kristek, et al., 2019; Kristek, et } \\
\text { al., 2019) }\end{array}$ & 2019 & $\begin{array}{l}\text { meta-análise } \\
\text { (nível de } \\
\text { evidência 1) }\end{array}$ & $\begin{array}{c}\text { o presente estudo in } \\
\text { vitro examinou se } \\
\text { uma dose da } \\
\text { mistura de } \\
\text { polifenol, extrato de } \\
\beta \text { - glucano ou farelo } \\
\text { de aveia digerido } \\
\text { como matriz } \\
\text { alimentar inteira } \\
\text { modula } \\
\text { beneficamente a } \\
\text { microbiota } \\
\text { intestinal }\end{array}$ & $\begin{array}{c}\text { a aveia aumentou bifidobactérias, ácido } \\
\text { acético e ácido propiônico, e, ao invés de } \\
\text { seus principais } \beta \text { - glucano ou polifenóis } \\
\text { bioativos. Por isso, a aveia como um } \\
\text { alimento integral causou o maior impacto } \\
\text { na microbiota }\end{array}$ \\
\hline $\begin{array}{l}\text { Comparative effects of different } \\
\text { whole grains and brans on } \\
\text { blood lipid: a network meta- } \\
\text { analysis (Hui, et al., 2019) }\end{array}$ & 2019 & $\begin{array}{l}\text { meta-análise } \\
\text { (nível de } \\
\text { evidência 1) }\end{array}$ & $\begin{array}{c}\text { o objetivo foi } \\
\text { estimar e classificar } \\
\text { os efeitos de } \\
\text { diferentes grãos } \\
\text { inteiros e farelos no } \\
\text { controle dos lipídios } \\
\text { do sangue } \\
\end{array}$ & $\begin{array}{l}\text { o farelo de aveia foi a intervenção mais } \\
\text { eficaz para melhorias de CT e LDL-C, } \\
\text { com diminuições significativas }\end{array}$ \\
\hline $\begin{array}{c}\beta \text {-glucans and cholesterol } \\
\text { (Review) (Sima, Vannucci, \& } \\
\text { Vetvicka, 2018) }\end{array}$ & 2018 & $\begin{array}{c}\text { revisão } \\
\text { sistemática (nível } \\
\text { de evidência 1) }\end{array}$ & $\begin{array}{c}\text { avaliar as } \\
\text { informações } \\
\text { atualizadas sobre } \\
\text { vários aspectos das } \\
\text { fibras alimentares e } \\
\beta \text {-glucanas na } \\
\text { regulação dos níveis } \\
\text { de colesterol } \\
\end{array}$ & $\begin{array}{l}\text { as } \beta \text {-glucana são as mais promissoras para } \\
\text { uso no tratamento de pacientes com } \\
\text { dislipidemia pois promovem um } \\
\text { reequilíbrio dos níveis de colesterol com } \\
\text { base fisiológica }\end{array}$ \\
\hline
\end{tabular}

Fonte: Autoria própria (2021).

Após as buscas os artigos encontrados foram classificados de acordo com Melnyk e Fineout-Overholt (2018) a qual seguiu os parâmetros de categorização do nível de evidências, indicados na Figura 2. 
Figura 2 - Níveis de evidência de estudos.

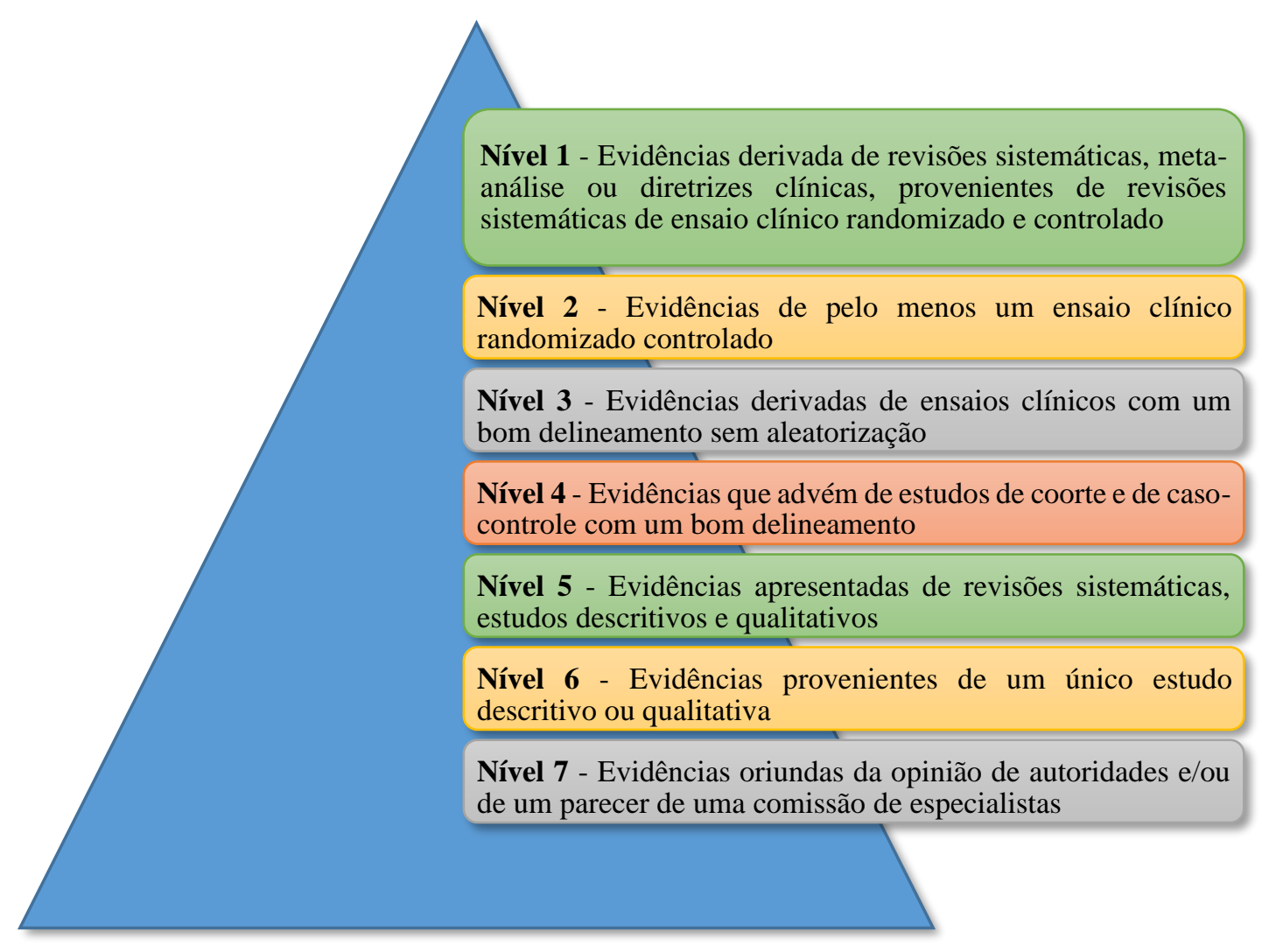

Fonte: Adaptada de Melnyk e Fineout-Overholt (2018).

\section{Discussão}

\subsection{Allium sativum}

A Allium sativum $L$ pertencente à família das Amaryllidaceae, sendo conhecida popularmente como alho. Ela é uma planta herbácea aromática utilizada como especiaria na culinária, bem como na medicina tradicional em povos antigos (Lima, Lima, Francisco, \& Amaral, 2020). O alho tem a capacidade de trazer benefícios à saúde em virtude de suas propriedades terapêuticas de ação antioxidante, hipoglicemiante, hipolipemiante, antitrombótica, antiapoptótica, antihipertensiva, antihipertrófica, antiarrítmica, agindo efetivamente na prevenção e tratamento de doenças de origem cardiovascular (Duque, Massolar, \& Junior, 2018).

De acordo com o estudo de El-Saber Batilha e seus colaboradores (2020), as preparações de alho possuem efeitos significativos nas dislipidemias em virtude do seu potencial redutor lipídico. A Allium sativum L é eficiente na diminuição dos níveis séricos de colesterol total ao dificultar a biossíntese do colesterol no fígado e ao excretar esteroides tanto ácidos como neutros, bem como é eficaz no controle de TG e na oxidação de LDL-c. Gyawali e companheiros ratificam que o consumo de alho reduz o colesterol total em 5\% e o LDL-c em 6\% em indivíduos com níveis elevados de colesterol total (CT médio> 200 $\mathrm{mg} / \mathrm{dl}$ ), sendo clinicamente relevante, já que está relacionado com a redução no risco de doenças coronárias e vasculares (Gyawali, Vohra, Orme-Johnson, Ramaratnam, \& Schneider, 2021).

Ademais, é válido ressaltar também que nos achados de Kirichenko e colaboradores (Kirichenko, et al., 2020) foi mencionado que o extrato de alho pode impossibilitar a atuação da enzima que ocasiona a formação do LDL de caráter aterogênico. Ademais, a oxidação da LDL é minimizada por conta dos efeitos terapêuticos ao agir como antioxidante e antitrombótico. Em contrapartida, nos estudos de Suances e colaboradores não foram encontradas diferenças significativas sobre 
a redução dos níveis de colesterol total, principalmente do LDL-c nos pacientes durante o estudo (Suances, Estévez, \& Estévez, 2018).

A ação antitrombótica do alho acontece devido à ocorrência da supressão da ciclooxigenase, uma enzima que participa da rota metabólica de conversão do ácido araquidônico em prostaglandinas a qual está responsável pela mediação do processo inflamatórios e do fluxo de sangue, da redução na síntese de tromboxano B2 e de vasoconstritores e, ainda, a administração efetiva das atividades de fosfolipases de membrana (Kirichenko, et al., 2020). Outrossim, o alho auxilia na redução da agregação plaquetária, já que aumenta a fibrinólise e diminui a viscosidade plasmática, sendo interessante o seu uso para o tratamento de pacientes com aterosclerose (Kirichenko, et al., 2020; Sobenin, Myasoedova, Iltchuk, Zhang, \& Orekhov, 2019).

\subsection{Linum usitatissimum $L$.}

Linun usitatissimun L., conhecido popularmente como linhaça, tem origem asiática e pertence à família das lináceas. É uma semente que consegue ser cultivada não apenas no clima quente, mas ainda no frio, sendo muito versátil, visto que pode ser empregada tanto para a formação de óleos, quanto de farelos, pelo seu grande teor de ômega-3 e fibras (Qamar, et al., 2019).

$\mathrm{Na}$ semente de linhaça, encontram-se quantidades relevantes de ácido graxo $\alpha$-linolênico (ômega-3), sendo a fonte vegetal que mais contêm este tipo de gordura, além de conter o ácido linoleico (ômega 6), sendo esses ácidos graxos poliinsaturados essenciais e importantes para a redução do risco de doenças cardiovasculares (Shekhara, Anurag, Prakruthi, \& Mahesh, 2020). A linhaça também apresenta fibras solúveis e insolúveis em sua composição; as solúveis agem no metabolismo lipídico, sendo relacionadas à redução do risco de dislipidemias. Já as insolúveis, promovem saciedade e agem no trânsito intestinal, diminuindo-o e, consequentemente, reduzindo a absorção das gorduras (Gonçalves, Pereira, \& Junior, 2020).

Conforme demonstrados nos resultados, duas revisões sistemáticas (Masjedi, Pour, Shokoohinia, \& Asgary, 2021; Hadi, et al., 2020) objetivaram avaliar pesquisas científicas sobre a eficiência da suplementação de linhaça e seus produtos na melhoria dos perfis lipídicos de adultos, congregando 76 ensaios clínicos randomizados e controlados realizados com indivíduos saudáveis e dislipidêmicos.

A metanálise de Hadi e colaboradores (2020) demonstrou redução nos níveis de colesterol por meio de suplementação de linhaça e seus derivados por período menor que 12 semanas em indivíduos dislipidêmicos, além de redução nos níveis de LDL-c a partir de 12 semanas de suplementação. A suplementação da linhaça inteira, seja de curta ou longa duração, propiciou a redução dos níveis de TG séricos, sem alteração do HDL-c.

Na revisão sistemática de Masjedi e colaboradores (2021) a análise do efeito de suplementação de linhaça em pessoas saudáveis no tocante aos níveis de colesterol total, LDL-c e HDL-c e TG permitiu verificar redução de, principalmente, colesterol total e aumento do HDL-c. A análise de subgrupos considerou fatores como: forma de consumo da linhaça, dose, duração e índice de massa corporal (IMC). Logo, observou-se redução no colesterol total apenas em pessoas com IMC $>25 \mathrm{~kg} / \mathrm{m} 2$, além de aumento no HDL-c no grupo com IMC $>25$ ou que recebeu uma pequena dose de linhaça ou utilizou a semente oleosa da linhaça. No tocante ao LDL-c, essa lipoproteína reduziu em estudos com indivíduos com IMC>25 ou que usaram a semente de linhaça não oleosa ou tenham recebido uma elevada dose de linhaça. Em indivíduos dislipidêmicos, os estudos demonstraram efeitos benéficos da linhaça no colesterol, HDL-c, LDL-c e TG. Na análise de subgrupos, considerou-se a forma de consumo de linhaça e duração dos estudos e foi possível concluir que a redução de colesterol, LDL-c e TG ocorreu em todos os subgrupos. Entretanto, a redução do HDL-c ocorreu apenas em subgrupos com duração maior que oito semanas e que utilizaram a linhaça não oleosa.

Ademais, a partir da análise de quatro ensaios clínicos (Skoczyńska, Gluza, Wojakowska, Turczyn, \& Skoczyńska, 2018) (Soltanian \& Janghorbani, 2019) (Haghighatsiar, Askari, Saraf-Bank, Feizi, \& Keshmiri, 2019) (Kanikowska, et al., 2020), com um total de 315 indivíduos participantes distribuídos em grupo intervenção e controle, que avaliaram o efeito da suplementação de óleo (Skoczyńska, Gluza, Wojakowska, Turczyn, \& Skoczyńska, 2018), pó e biscoito (Soltanian \& 
Janghorbani, 2019) (Kanikowska, et al., 2020) de linhaça sobre o perfil lipídico de indivíduos dislipidêmicos (Skoczyńska, Gluza, Wojakowska, Turczyn, \& Skoczyńska, 2018) (Haghighatsiar, Askari, Saraf-Bank, Feizi, \& Keshmiri, 2019) (Kanikowska, et al., 2020), observou-se os efeitos benéficos da linhaça ao propiciar uma redução significante nos níveis de colesterol (Soltanian \& Janghorbani, 2019) (Haghighatsiar, Askari, Saraf-Bank, Feizi, \& Keshmiri, 2019) (Kanikowska, et al., 2020), TG (Skoczyńska, Gluza, Wojakowska, Turczyn, \& Skoczyńska, 2018) (Haghighatsiar, Askari, Saraf-Bank, Feizi, \& Keshmiri, 2019), LDL-c (Skoczyńska, Gluza, Wojakowska, Turczyn, \& Skoczyńska, 2018) (Soltanian \& Janghorbani, 2019) (Haghighatsiar, Askari, Saraf-Bank, Feizi, \& Keshmiri, 2019) (Kanikowska, et al., 2020), além do aumento de HDL-c

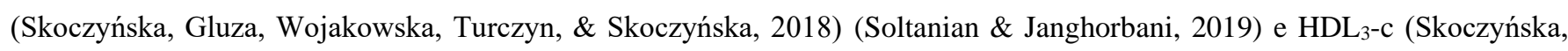
Gluza, Wojakowska, Turczyn, \& Skoczyńska, 2018), sendo demonstrados efeitos duradouros após 4 semanas do fim do estudo (Skoczyńska, Gluza, Wojakowska, Turczyn, \& Skoczyńska, 2018).

Os resultados obtidos nesses estudos corroboram o estudo de Islam, Ahmed, Ahsan e Lee (Islam, Ahmed, Ahsan, \& Lee, 2021), que esclareceu acerca dos mecanismos moleculares produzidos pelas fibras dietéticas, a exemplo da linhaça, no perfil lipídico ao promoverem a redução da captação dos ácidos biliares no intestino delgado e maior excreção biliar no conteúdo fecal. Além de propiciarem menor absorção de colesterol dietético devido ao aumento do volume e viscosidade do bolo alimentar, bem como redução na biossíntese de colesterol em virtude da produção de ácidos graxos de cadeia curta por fermentação no intestino grosso, dentre os quais cita-se o propionato que estimula a liberação do polipeptídio YY (PYY) e do peptídeo semelhante ao glucagon 1 (GLP-1) os quais contribuem para a redução na concentração de LDL-c plasmático.

O estudo de Parikh, Netticadan e Pierce (2018) demonstrou o mecanismo de ação do ácido linolênico (ômega-3) presente na linhaça sobre o perfil lipídico, haja vista que esse lipídeo é metabolizado em ácido eicosapentaenoico (EPA) e ácido docosaexaenoico (DHA), os quais atuam na ativação de receptores ativados por proliferadores de peroxissoma gama (PPAR- $\gamma$ ) que estão envolvidos em atividades anti-inflamatórias e anti-aterogênicas, contribuindo para a redução do estresse oxidativo.

\subsection{Avena sativa}

A Avena sativa pertence à família das Poaceae, possuindo o nome popular de aveia. Ela é utilizada principalmente como fonte de fibras solúveis, proteínas e minerais, entretanto, possui diversas outras funções derivadas dos ácidos fenólicos, polifenóis e terpenos, que auxiliam desde sintomas cardiovasculares, como redução do colesterol, até manifestações psicotrópicas, como insônia e ansiedade, sendo aquela que apresentou resultados mais eficazes na melhora dos níveis de TG e LDL-c, dentre outros grãos, como cevada, arroz integral, trigo e farelo de trigo (Trautwein \& McKay, 2020; Hui, et al., 2019; Kennedy, et al., 2020; Borges, et al., 2021).

A eficácia da A. sativa no tratamento da dislipidemia pode ser encontrada em seus diversos compostos, em especial nas beta-glucanas, onde identificou-se uma relação inversamente proporcional ao consumo desse composto à concentração de LDLc. Registrou-se que a ingestão de 3,5g/dia da beta-glucana da aveia reduziu 4,2\% dos níveis de LDL-c, e 4,8\% de não-HDL-c, em comparação com o grupo controle, e outro teste indicou redução de $6 \%$ de LDL-c quando ingerido 5,1g/dia da beta-glucana (Trautwein \& McKay, 2020; Cicero, et al., 2020).

Apesar de não se saber exatamente como ocorre, acredita-se estar relacionado com sua capacidade de aumentar a viscosidade, que interfere na captação dos ácidos biliares, diminuindo a quantidade de colesterol absorvido no intestino delgado, a qual impacta a quantidade de quilomícrons absorvidos e, consequentemente, ameniza o teor de colesterol circulante. Além disso, a habilidade das fibras solúveis, como as betas-glucanas, de aumentar a fermentação no cólon e produzir ácidos graxos de cadeia curta auxilia na normalização do perfil lipídico que diminui o colesterol sanguíneo de forma indireta por inibir a síntese de colesterol no fígado (Ferreira, et al., 2021; He, et al., 2018). 
Outrossim, as beta-glucanas podem modificar a microbiota intestinal aumentando a diversidade microbiana como Bacteroidaceae e Bacteroidetes/Firmicutes (Hui, et al., 2019).. Dessa forma, afetam também o sistema imune reparando inflamações crônicas e reduzindo citocinas pró-inflamatórias, além de promover propriedades anti-infecciosas e anticancerígenas das defesas humoral e celular (Sima, Vannucci, \& Vetvicka, 2018).

\section{Conclusão}

O uso terapêutico das plantas medicinais é uma prática tradicional de gerações e foi respaldada legalmente para ser utilizada por profissionais capacitados na assistência à saúde, visto que os fitoterápicos necessitam de pesquisa e comprovações científicas para que a prescrição possua eficácia, qualidade e segurança. Assim, ao empregar o uso dessas plantas em doenças crônicas não transmissíveis, o estudo em questão considerou os efeitos de alguns fitoterápicos no tratamento das dislipidemias, em especial: o alho cujo efeito está principalmente ligado ao controle dos níveis sanguíneos de TG e LDL-c ; a linhaça que dependendo da forma e tempo de uso se mostrou eficaz na redução do LDL-c, dos níveis de TG séricos e de colesterol, da mesma forma que proporciona o aumento do HDL-c; e a aveia, cuja eficácia está ligada às beta-glucanas, mostrando ação na diminuição dos níveis de LDL-c e de não-HDL-c. Por meio dessas ações, essas plantas sinalizam ser ótimos cardioprotetores.

Ademais, é válido visar as ações corretivas adequadas, como promover a mudança no comportamento dietético, a fim de controlar a prevalência de fatores de risco cardiovascular na população. Logo, com base na metodologia empregada e nos resultados encontrados, é possível proferir que os fitoterápicos pesquisados apresentaram resultados satisfatórios no tratamento da dislipidemia. Entretanto, mais estudos precisam ser realizados para consolidar os efeitos positivos dessas plantas no manejo terapêutico das dislipidemias.

\section{Referências}

Batiha, G. E., Beshbishy, A. M., Wasef, L. G., Elewa, Y. H., Al-Sagan, A. A., El-Hack, M. E., \& Devkota, H. P. (2020). Chemical Constituents and Pharmacological Activities of Garlic (Allium sativum L.): A Review. Nutrients, 12(3), 872.

Bezerra, G. A., Oliveira, L. d., Vilar, M. S., Confessor, M. V., \& Vilar, D. d. (2017). Plantas medicinais com efeitos terapêuticos para distúrbios metabólicos.

Borges, A. C., Rodrigues, B. F., Pires, L. S., Rodrigues, G. S., Santos, I. C., Santos, G. B., . . Veloso, R. B. (2021). Dislipidemia mista e o risco da evolução de doenças cardiovasculares em idosos. Research, Society and Development, 10(3), e38310313416.

Camargo, J. S., Zamarchi, T. B., Balieiro, A. A., Pessoa, F. A., \& Camargo, L. M. (2021). Prevalência de obesidade, pressão arterial elevada e dislipidemia e seus fatores associados em crianças e adolescentes de um município amazônico. Rev Bra Cresc e Desv Hum, 31(1), 37-46. doi:10.36311/jhgd.v31.11209

Choudhary, P. R., Jani, R. D., \& Sharma, M. S. (julho de 2018). Effect of Raw Crushed Garlic (Allium sativum L.) on Components of Metabolic Syndrome. J Diet Suppl, 15(4), 499-506.

Cicero, A. F., Fogacci, F., Veronesi, M., Strocchi, E., Grandi, E., Rizzoli, E., . . Borghi, C. (2020). A Randomized Placebo-Controlled Clinical Trial to Evaluate the Medium-Term Effects of Oat Fibers on Human Health: The Beta-Glucan Effects on Lipid Profile, Glycemia and inTestinal Health (BELT) Study. Nutrients, 12(3), 686-697.

Duque, A. P., Massolar, C. d., \& Junior, L. F. (2018). Propriedades cardioprotetoras do alho (Allium sativum). Revista Brasileira de Plantas Medicinais, 20, 7182 .

Elias, C. d., Silva, L. A., Martins, M. T., Ramos, N. A., Souza, M. d., \& Hipólito, R. L. (2012). Quando chega o fim? Uma revisão narrativa sobre terminalidade do período escolar para alunos deficientes mentais. Revista Electrónica em Saúde Mental, Álcool e Drogas, pp. 48-53.

Evangelista, K. C., Leite, L. D., \& Lima, S. C. (2013). Doenças Cardiovasculares. Em S. M. Cozzolino, \& C. Cominetti, Bases Bioquímicas e Fisiológicas da Nutrição: nas diferentes fases da vida, na saúde e na doença. (pp. 811-853). Baurú SP: Manole.

Ferreira, R. d., Mendonça, L. A., Santos, C. d., Hiane, P. A., Matias, R., Franco, O. L., . . Guimarães, R. d. (2021). Do Bioactive Food Compound with Avena sativa L., Linum usitatissimum L. and Glycine max L. Supplementation with Moringa oleifera Lam. Have a Role against Nutritional Disorders? An Overview of the In Vitro and In Vivo Evidence. Nutrients, 13(7), 2294-2316.

Gonçalves, J. d., Pereira, A. D., \& Junior, A. J. (2020). Linhaça como fitoterápico na dislipidemia - Uma revisão bibliográfica. Revista Científica Multidisciplinar Núcleo do Conhecimento, 01(04), 55-87.

Gyawali, D., Vohra, R., Orme-Johnson, D., Ramaratnam, S., \& Schneider, R. H. (2021). A Systematic Review and Meta-Analysis of Ayurvedic Herbal Preparations for Hypercholesterolemia. Medicina, 57(6), 546-570. 
Hadi, A., Askarpour, M., Salamat, S., Ghaedie, E., Symonds, M. E., \& Miraghajani, M. (2020). Effect of flaxseed supplementation on lipid profile: an updated systematic review and dose-response meta-analysis of sixty-two randomized controlled trials. Pharmacological Research.

Haghighatsiar, N., Askari, G., Saraf-Bank, S., Feizi, A., \& Keshmiri, H. (2019). Effect of Flaxseed Powder on Cardiovascular Risk Factor in Dyslipidemic and Hypertensive Patients. Int J Prev Med, 10(218).

He, C., Fu, P., Zhang, K., Xia, Q., Yang, Y., \& Xie, L. (2018). Chinese herbal medicine for dyslipidemia: protocol for a systematic review and meta-analysis. Medicine (Baltimore), 97(44), p. e13048.

Hui, S., Liu, K., Lang, H., Liu, Y., Wang, X., Zhu, X., \& Mi, M. (2019). Comparative effects of different whole grains and brans on blood lipid: a network metaanalysis. Eur J Nutr, 58, 2779-2787.

Islam, S. U., Ahmed, M. B., Ahsan, H., \& Lee, Y. S. (2021). Recent Molecular Mechanisms and Beneficial Effects of Phytochemicals and Plant-Based Whole Foods in Reducing LDL-C and Preventing Cardiovascular Disease. Antioxidants (Basel), 10(5), 784-812.

Kanikowska, D., Korybalska, K., Mickiewicz, A., Rutkowski, R., Kuchta, A., Sato, M., \& Witowski, J. (2020). Flaxseed (Linum Usitatissimum L.) Supplementation in Patients Undergoing Lipoprotein Apheresis for Severe Hyperlipidemia-A Pilot Study. Nutrients, 12(4), $1137-1147$.

Kennedy, D. O., Bonnländer, B., Lang, S. C., Pischel, I., Forster, J., Khan, J., \& Wightman, E. L. (2020). Acute and Chronic Effects of Green Oat (Avena sativa) Extract on Cognitive Function and Mood during a Laboratory Stressor in Healthy Adults: A Randomised, Double-Blind, Placebo-Controlled Study in Healthy Humans. Nutrients, 12(6), 1598-1617.

Kirichenko, T. V., Sukhorukov, V. N., Markin, A. M., Nikiforov, N. G., Liu, P. Y., Sobenin, I. A., \& Aliev, G. (2020). Medicinal Plants as a Potential and Successful Treatment Option in the Context of Atherosclerosis. Front Pharmacol, 8(11), 403.

Kristek, A., Wiese, M., Heuer, P., Kosik, O., Schär, M., Soycan, G., \& Spencer, J. (2019). Oat bran, but not its isolated bioactive $\beta$-glucans or polyphenols, have a bifidogenic effect in an in vitro fermentation model of the gut microbiota. Br J Nutr, 121(5), pp. 549-559.

Lima, I. B., Lima, R. O., Francisco, R. C., \& Amaral, C. S. (2020). Inovação na prevenção de doenças cardiovasculares a partir da alimentação saudável. Rev Bras Crescimento Desenvolv Hum, 6(10), 80508-80525.

Masjedi, M. S., Pour, P. M., Shokoohinia, Y., \& Asgary, S. (2021). Efeitos da semente de linhaça sobre os lipídios do sangue em indivíduos saudáveis e dislipidêmicos: uma revisão sistemática e meta-análise de ensaios clínicos randomizados. Curr Probl Cardiol.

Medina, M. C. (2017). Efecto de la mezcla de cereales con extracto acuoso de Ananás comosus en los niveles séricos de colesterol y triglicéridos de personas con dislipidemia. Sciéndo, 20(2), 71-81.

Melnyk, B., \& Fineout-Overholt, E. (2018). Evidence-based practice in nursing and healthcare: A guide to best practice (4a ed.). Philadelphia: Wolters Kluwer Health.

Ministério da Saúde. (2006). Departamento de Atenção Básica. Política Nacional de Práticas Integrativas e Complementares no SUS - PNPIC-SUS. Brasília: Ministério da Saúde.

Ministério da Saúde. (2017). Vigitel Brasil 2016 Saúde Suplementar: vigilância de fatores de risco e proteção para doenças crônicas por inquérito telefônico (1ª ed.). Brasília: Ministério da Saúde.

Nogueira-de-Almeida, C. A., de Mello, E. D., de Mello, P. P., de Mello, P. D., Zorzo, R. A., \& Ribas Filho, D. (2017). Consenso da Associação Brasileira de Nutrologia sobre manejo da dislipidemia secundária à obesidade infanto-juvenil. International Journal of Nutrology, 10(4), 161-178.

Oliveira, L. B., Carvalho, I. B., Dourado, C. S., Dourado, J. C., \& Nascimento, M. O. (2017). Prevalência de dislipidemias e fatores de risco associados. J. Health Biol. Sci., 5(4), 320-325.

Parikh, M., Netticadan, T., \& Pierce, G. N. (2018). Flaxseed: its bioactive components and their cardiovascular benefits. Am J Physiol Heart Circ Physio, H146H159.

Précoma, D. B., Oliveira, G. M., SIMÃO, A. F., Dutra, O. P., Coelho, O. R., \& Izar, C. O. (2019). Atualização da diretriz de prevenção cardiovascular da Sociedade Brasileira de Cardiologia - 2019. Arq Bras Cardiol, 113(4), 787-891. doi:10.5935/abc.20190204

Qamar, H., Ilyas, M., Shabbir, G., Irshad, G., Nisar, F., Abbas, S. M., \& Arshad, A. (2019). Flax: Ancient to modern food. Pure Appl. Biol., 8(4), 2227-2269.

Raymond, L. J., \& Couch, S. C. (2018). Dietoterapia para as Doenças cardiovasculares. Em L. K. Mahan, \& L. J. Raymond, Krause Alimentos, Nutrição e Dietoterapia. São Paulo: Editora Roca.

Rother, E. T. (2007). Revisão sistemática X revisão narrativa. Acta paulista de enfermagem.

Santana, M. D., Sá, J. S., Neves, A. F., Figueredo, P. G., \& Viana, J. A. (2018). O Poder das Plantas Medicinais: uma Análise Histórica e Contemporânea sobre a Fitoterapia na visão de Idosas. Multidebates, 2(2), 10-27.

Shekhara, N., Anurag, A. P., Prakruthi, M., \& Mahesh, M. S. (2020). Flax Seeds ( Linum usitatissimmum): Nutritional composition and health benefits. IP Journal of Nutrition, Metabolism and Health Science, 3(2), 35-40.

Sima, P., Vannucci, L., \& Vetvicka, V. (2018). ß-glucans and cholesterol (Review). Int J Mol Med, 41(4), $1799-1808$.

Skoczyńska, A. H., Gluza, E., Wojakowska, A., Turczyn, B., \& Skoczyńska, M. (2018). Linseed oil increases HDL3 cholesterol and decreases blood pressure in patients diagnosed with mild hypercholesterolaemia. Kardiol Pol, 76(8), 1242-1250. 
Research, Society and Development, v. 11, n. 3, e22311326395, 2022

(CC BY 4.0) | ISSN 2525-3409 | DOI: http://dx.doi.org/10.33448/rsd-v11i3.26395

Sobenin, I. A., Myasoedova, V. A., Iltchuk, M. I., Zhang, D. W., \& Orekhov, A. N. (2019). Therapeutic effects of garlic in cardiovascular atherosclerotic disease. Chin J Nat Med, 17(10), 721-728.

Soltanian, N., \& Janghorbani, M. (2019). Effect of flaxseed or psyllium vs. placebo on management of constipation, weight, glycemia, and lipids: A randomized trial in constipated patients with type 2 diabetes. Clin Nutr ESPEN, 29, 41-48.

Souza, N. A., Vieira, S. A., Fonsêca, P. C., Andreoli, C. S., Priore, S. E., \& Franceschini, S. d. (2019). Dislipidemia familiar e fatores associados a alterações no perfil lipídico em crianças. Cien Saude Colet [online], 24(1), pp. 323-332.

Suances, A. E., Estévez, A. E., \& Estévez, M. E. (2018). Estudio experimental y controlado en farmacia comunitaria: influencia de la administración de un complemento alimenticio de ajo en la evolución de la hipercolesterolemia. Farmacéuticos comunitarios, 10(1), 33-40.

Teixeira, M. E., Vitorino, P. V., Amodeo, C., Martinez, T., Brandão, A. A., Barbosa, E. C., \& Barroso, W. K. (2021). Fatores de Risco Cardiovascular em Cardiologistas Especialistas pela Sociedade Brasileira de Cardiologia. Arq. Bras. Cardiol, 116, 774-81.

Trautwein, E. A., \& McKay, S. (2020). The Role of Specific Components of a Plant-Based Diet in Management of Dyslipidemia and the Impact on Cardiovascular Risk. Nutrients, 12(9), 2671-2692.

Wlosinska, M., Nilsson, A. C., Hlebowicz, J., Malmsjö, M., Fakhro, M., \& Lindstedt, S. (2019). Aged garlic extract preserves cutaneous microcirculation in patients with increased risk for cardiovascular diseases: A double-blinded placebo-controlled study. Int Wound J, 16(6), 1487-1493.

Wlosinska, M., Nilsson, A.-C., Hlebowicz, J., Fakhro, M., Malmsjö, M., \& Lindstedt, S. (2021). Aged Garlic Extract Reduces IL-6: A Double-Blind PlaceboControlled Trial in Females with a Low Risk of Cardiovascular Disease. Evid Based Complement Alternat Med, 31, 6636875. 